\title{
Breastfeeding Duration in a Multiethnic Population in Hawaii
}

\author{
Rachel Novotny, PhD, RD, Mya Moe Hla, MBBS, MPH, PhD, Edith C. Kieffer, PhD, MPH, \\ Chai-Bin Park, MD, DrPH, Joanne Mor, MS, and Maureen Thiele, MPH, RD
}

\begin{abstract}
Background: The increasing ethnic diversity in the United States necessitates a study of variations in infant feeding patterns among ethnic groups. This study was conducted as part of Hawaii's surveillance system to identify infant feeding patterns in Hawaii; specifically, to identify factors influencing duration of breastfeeding among ethnically diverse mothers. Methods: All women who delivered an infant in Hawaii between January 1 and March 31, 1989, were mailed surveys 14 to 19 months after delivery. Fiftyone percent $(\mathrm{n}=2011)$ of women responded, of whom $1574(78 \%)$ did some breastfeeding and are included in the analysis of prediction of weaning (cessation of breastfeeding). Cox regression (survival) analysis was used to predict weaning. Results: The median duration of breastfeeding was 150 days; 45 percent of infants were still breastfeeding at age 6 months and 16 percent at age 1 year. Factors associated with early weaning were Japanese ethnicity; mother born in a country other than the United States, Japan, or the Philippines; first language other than English, or two languages at home; employed full-time outside the home; introduced formula or fruit before age 6 months; received formula from the WIC program; and stopped breastfeeding for convenience, breast problems, problems getting breastfeeding started, insufficient milk, baby refusing the breast, and a sick baby. Factors associated with late weaning were older maternal age; college education; living on a rural island; previous breastfeeding experience; helpful breastfeeding advice from family or friends; receiving WIC for breastfeeding mothers; introducing the cup before age 6 months; and not giving fruit to the baby. Conclusion: In Hawaii, programs that address how and when to introduce foods, use of formula, and management of outside employment and breastfeeding should be made available to those groups of women at risk for early weaning to lengthen their duration of breastfeeding. (BIRTH 27:2 June 2000)
\end{abstract}

Rachel Novotny is Associate Professor at the Department of Food Science and Human Nutrition, College of Tropical Agriculture and Human Resources, University of Hawaii at Manoa, Honolulu, Hawaii. Mya Moe Hla is Assistant Professor, Chai-Bin Park is Professor, and Joanne Mor is Computer Specialist at the School of Public Health, University of Hawaii at Manoa, Honolulu, Hawaii. Edith Kieffer is Assistant Professor at the School of Public Health, University of Michigan, Ann Arbor, Michigan. Maureen Thiele is Nutritionist at the Hawaii State Department of Health, Hilo, Hawaii.

This project was funded by the Nutrition Surveillance Program, Department of Health, Honolulu, Hawaii.

Address correspondence to Rachel Novotny, Department of Food Science and Human Nutrition, College of Tropical Agriculture and Human Resources, University of Hawaii at Manoa, Honolulu, HI 96822.

(C) 2000 Blackwell Science, Inc.
Investigations of breastfeeding patterns and their determinants should be conducted so that health caregivers can devise strategies to increase breastfeeding and achieve the national health objectives. Year 2000 national health objectives recommend that at least 50 percent of infants should continue breastfeeding until 6 months of age (1). Recent recommendations by the American Academy of Pediatrics urge that mothers should continue to breastfeed throughout the infant's first year of life (2).

The increasing ethnic diversity in the United States necessitates a study of variations in infant feeding patterns among ethnic groups. Our previous studies identified influences on the decision to breastfeed but not factors influencing the duration of breastfeeding 
(3-6). Other studies examined duration of breastfeeding in different populations (7-9), but research that has included Japanese Americans or native Hawaiians has categorized them either in one group of Asian (10) or in another group of Asian and Pacific Islander (11). The objective of this study was to examine factors influencing duration of breastfeeding among Caucasian, Japanese, Filipino, Hawaiian/part-Hawaiian, and other ethnicities of women in Hawaii.

\section{Methods}

The study population was drawn from all live births in the state of Hawaii between January 1 and March 31, 1989, and data were obtained from the state of Hawaii Vital Records Registry. Of the 4498 questionnaires mailed, 585 were not deliverable (change of address), leaving 3913 questionnaires that were delivered. After two reminder postcards were sent, responses were received from 2011 mothers (response rate of $51 \%$ ).

The questionnaire was designed to describe infant feeding patterns in the state of Hawaii, and concerned factors influencing initiation and duration of infant feeding practices (12). Data were obtained retrospectively when the infant was age 14 to 19 months. Participants could respond to the survey in days, weeks, or months; all data were converted to days for analysis.

\section{Analysis of Data}

Analysis was done using SAS software (13), and a probability level of $p<0.05$ was considered significant. The key dependent variable was the infant's age at cessation of breastfeeding. At the time when data were gathered, 157 censored infants were still breastfeeding and were included in the analysis.

Since not all women had completed breastfeeding at the time of the survey, the Cox Proportional Hazard Model, Kaplan Meir Procedure $(13,14)$, was used to calculate "risk" of cessation of breastfeeding (weaning), using data on breastfeeding duration. Risk factors for stopping breastfeeding are presented as hazard ratios with 95 percent confidence intervals. Hazard ratios were adjusted for other variables in the model.

Independent variables that were considered to have a possible effect on duration of breastfeeding were used in the analysis. They included 1) participation in the supplementary nutrition program for Women, Infants and Children (WIC) for breastfeeding women or for formula-feeding infants; 2) college education, including any amount of college, and no college was the reference group; 3) ethnicity for which Caucasian ethnicity was the reference group (Japanese, Filipino, Hawaiian/part-Hawaiian, and other ethnicities); 4) marital status; 5) employment outside the home (fullor part-time); 6) attending school; 7) rural island residency (any island other than Oahu); 8) mother's age $(<35 \mathrm{yr}$ or $>35 \mathrm{yr}) ; 9)$ ages at which cereal, fruit, and formula were given (in days); 10) age at which the cup was introduced; 11) country of maternal birth; 12) language(s) spoken at home; 13) reasons for stopping breastfeeding; 14) previous breastfeeding experience (exclusive, mixed), if second birth order or higher; and 15) source of helpful advice on breastfeeding.

\section{Results}

At the time of the survey infants ranged in age from 419 to 564 days (mean $=472$ days $[16 \mathrm{mo}]$ ). Mothers ranged in age from 16 to 52 years (mean $=29.5 \mathrm{yr}$ ). Forty-one percent of the women were employed fulltime outside the home, 16 percent part-time, and 42 percent were not working outside the home. The mean age of the baby when women returned to work was 134 days $(5 \mathrm{mo})$. Twenty-seven percent of the original study population had participated in WIC. Other participant characteristics are described in Table 1.

At hospital discharge, 78 percent of mothers were breastfeeding, 50 percent exclusively and 28 percent combined breast and formula feeding. Those who breastfed, even a little, comprised the study population for this study. Of these women, 39 percent were Caucasian, 20 percent were Japanese, 12 percent were Fili-

Table 1. Characteristics of the Study Population $(n=$ 2011)

\begin{tabular}{lr}
\hline Characteristic & No. $(\%)$ \\
\hline Mother's educational achievement & \\
Some high school & $139(7)$ \\
Graduate high school & $589(30)$ \\
Some college & $581(29)$ \\
Graduated college & $477(24)$ \\
Graduate school & $192(10)$ \\
Mother's ethnicity & \\
Caucasian & $618(31)$ \\
Hawaiian/part-Hawaiian & $433(22)$ \\
Japanese & $288(15)$ \\
Filipino & $241(12)$ \\
Mixed & $213(11)$ \\
Chinese & $76(4)$ \\
Hispanic & $32(2)$ \\
Black & $29(2)$ \\
Other Asian & $26(1)$ \\
Pacific Islander & $17(1)$ \\
Mother's island of residence & \\
Oahu & $1396(69)$ \\
Hawaii (Big Island) & $265(14)$ \\
Maui County (Maui, Molokai, Lanai) & $176(9)$ \\
Kauai & $88(4)$ \\
Moved from Hawaii & $70(4)$ \\
\hline
\end{tabular}


pino, 14 percent were Hawaiian/part-Hawaiian, and 16 percent were other ethnicity. Sixty-eight percent had some college education, 7 percent were students, and 58 percent were employed full- or part-time at the time of the survey.

Of infants who breastfed at all, 77 percent were still breastfeeding at age 2 months, 45 percent at 6 months, and 16 percent at 1 year. In Fig. 1, the Kaplan Meir Procedure indicates that infants who were formula fed were likely to stop breastfeeding earlier than those who never formula fed or than those who started formula feeding later.

Risk factors for early weaning were Japanese ethnicity; mother born in a country other than the United States; first language other than English, or two languages at home; employed full-time outside the home; introduction of formula (the earlier formula was introduced, the higher the risk); receiving fruit before age 6 months; receiving formula from the WIC program; and stopping breastfeeding for convenience, breast problems, problems getting breastfeeding started, insufficient milk, baby refusing the breast, and a sick baby (Table 2).

Factors associated with longer duration of breastfeeding were older maternal age; college education; living on a rural island; previous breastfeeding experience; helpful breastfeeding advice from family

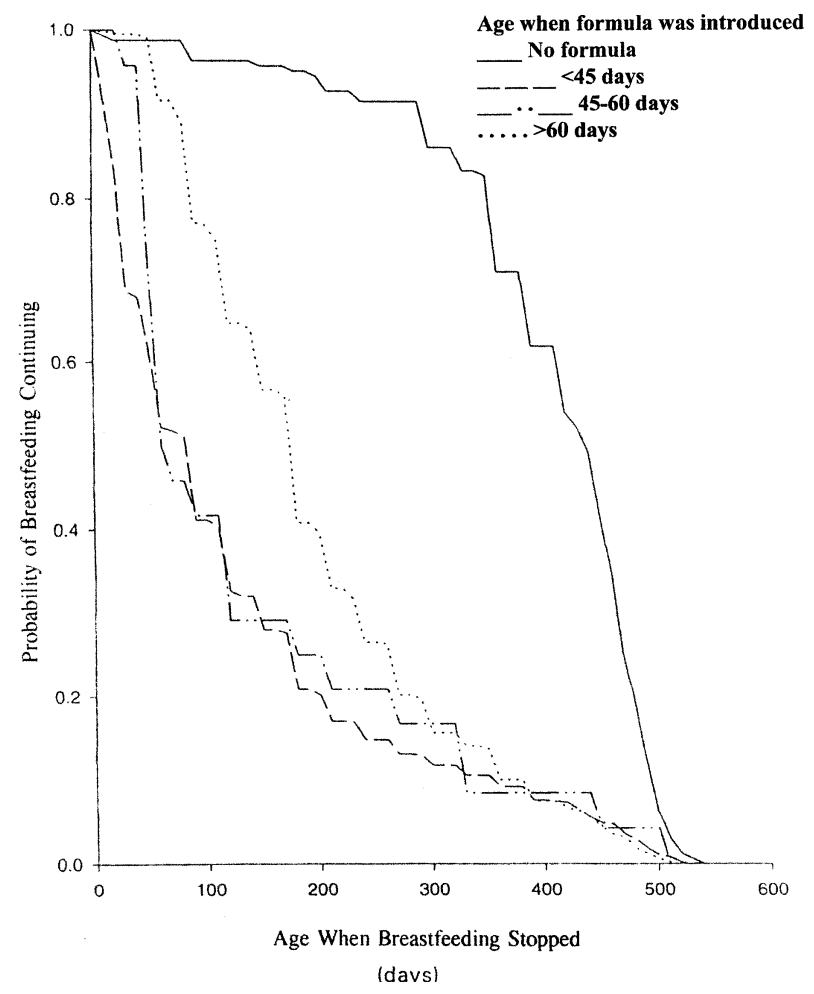

Fig. 1. Survival curve of breastfeeding cessation by formula use, Hawaii 1990 ( $\mathrm{n}=1478)$. or friends; receiving WIC for breastfeeding mothers; introducing the cup before age 6 months; and not giving fruit to the baby (Table 3). Receiving advice from the mother did not reach significance (data not shown).

\section{Discussion and Conclusions}

In Hawaii, 45 percent of infants were still breastfeeding at age 6 months, which is longer than in much of the United States (estimated at 20\%) (15). This duration is still below the national health objectives for the year 2000 (1).

The role of maternal birth on infant feeding practices in the Philippines was reported in a previous paper (5). Highly educated Philippines-born Filipino women were more likely to combine breastfeeding and formula feeding or to formula feed at hospital discharge than United States-born Filipinos or other ethnic groups in Hawaii. In a study of breastfeeding duration conducted in the Philippines, strong correlates of weaning included advice from health professionals that mothers should wean and receiving free formula samples.

In the current study Filipino women who breastfed were at higher risk for early weaning than other ethnic groups, although this finding was masked by including the variable of other or mixed language used in the home in the model (Table 2). Immigrants, especially, may be confused by the United States media exposure of the use of breasts for erotic purposes more than for breastfeeding (16), and media messages in their home countries implying that formula is better than human milk. Both factors may lead them to stop breastfeeding.

Perhaps the most surprising finding was that although Japanese women were likely to initiate breastfeeding (87\%) (12), they weaned earlier than other women, even after controlling for employment outside the home. Japanese women in Hawaii are a highly educated group and therefore this finding is at odds with the finding of increased duration of breastfeeding among women of higher socioeconomic status, which is more common. Further exploration of barriers to longer breastfeeding among Japanese women is warranted.

Introducing fruit (a common first food), and especially formula, was highly associated with weaning in this study. Although introduction of food is recommended at age 6 months, it should complement (or add to) breastmilk, not replace it $(2,17)$. The infant is less hungry after eating food or formula, thus initiating a cycle whereby decreased nursing leads to decreased breastmilk production (due to decreased stimulation of milk-producing hormones), and hence, increased food consumption and less breastfeeding. Breastmilk should remain the infant's key food until at least 1 
year of age. In Hawaii, most foods were introduced earlier (112 days) than is recommended (4), which contributed to early weaning (18). Another study conducted in Canada also reported that late introduction of solids was related to longer duration of breastfeeding (9).

Formula is nutritionally unnecessary if the infant is breastfeeding, and its introduction is a well-documented correlate of breastfeeding cessation (10,19-21). Reasons include possible nipple confusion by the infant (22), the relative ease of sucking from a bottle, and the mother's desire to replace breastfeeding with formula feeding (23). In addition, early introduction of food or formula may be a proxy for a perceived insufficiency of breastmilk $(9,18)$.

Formula may also be introduced as part of an intentional plan to stop breastfeeding (i.e., weaning). A previous study reported that reasons for stopping breastfeeding could be classified as intentional reasons about 33 percent of the time (return to paid employment, it was the "right time," for "convenience") and as unintentional reasons 34 percent of the time ("not enough milk," "infant refused the breast," mother or infant was ill," "breast problems," " "problems getting started," "mother pregnant"); the remaining reasons were classified as "other" (12). These data, however, included women who stopped breastfeeding when the infant was at different ages. Many unintentional reasons listed occurred as a result of formula feeding.

We can identify a time-associated phenomenon whereby formula introduction increased the risk of breastfeeding cessation (Fig. 1). Whether or not this breastfeeding cessation associated with formula feeding was an intentional or a biologic consequence cannot be known from these data.

Although the introduction of formula is time depen-

Table 2. Risk Factors for Early Cessation of Breastfeeding $(n=1567)$

\begin{tabular}{lrr}
\hline Risk Factor & Hazard Ratio & $95 \%$ CI \\
\hline Japanese ethnicity & 1.19 & $1.01-1.40$ \\
Other language spoken at home & 1.45 & $1.13-1.87$ \\
Mixed languages spoken at home & 1.79 & $1.38-2.32$ \\
Received WIC formula & 1.21 & $1.00-1.46$ \\
Works full-time & 1.34 & $1.10-1.64$ \\
Introduced formula <45 days & 4.29 & $3.43-5.37$ \\
Introduced formula 45-60 days & 4.01 & $2.50-6.45$ \\
Introduced formula > 60 days & 2.67 & $2.14-3.34$ \\
Introduced fruit < 6 mo & 1.20 & $1.07-1.34$ \\
Stopped breastfeeding because: & & \\
Not enough milk & 7.10 & $5.82-8.66$ \\
To go to school or work & 7.04 & $5.79-8.56$ \\
For convenience & 5.44 & $4.24-6.98$ \\
Had problems with breasts & 15.48 & $11.75-20.40$ \\
Had problems starting breastfeeding & 40.12 & $25.17-63.97$ \\
Baby was sick & 5.47 & $4.22-7.08$ \\
Baby refused the breast & 3.63 & $2.95-4.47$ \\
Other problems & 3.27 & $2.52-4.25$ \\
\hline
\end{tabular}

WIC $=$ supplemental nutrition program for Women, Infants and Children.

Table 3. Factors That Promote Longer Duration of Breastfeeding $(n=1567)$

\begin{tabular}{|c|c|c|}
\hline Risk Factor & Hazard Ratio & $95 \% C I$ \\
\hline Mother's age > $35 \mathrm{yr}$ & 0.71 & $0.62-0.82$ \\
\hline Mother college educated & 0.78 & $0.68-0.89$ \\
\hline Mother lives on rural island & 0.83 & $0.72-0.94$ \\
\hline Mother has previous breastfeeding experience & 0.81 & $0.71-0.93$ \\
\hline Mother has previous mixed feeding experience & 0.86 & $0.74-1.00$ \\
\hline Mother received WIC for breastfeeding mothers & 0.53 & $0.40-0.71$ \\
\hline Infant received no fruit before age 6 mo & 0.55 & $0.37-0.81$ \\
\hline Infant started cup before age 6 mo & 0.51 & $0.48-0.62$ \\
\hline Mother received helpful advice from family* & 0.72 & $0.52-0.98$ \\
\hline Mother received helpful advice from friends & 0.75 & $0.57-0.98$ \\
\hline
\end{tabular}

* Mother-in-law, father, baby's father, other female relative.

WIC $=$ supplemental nutrition program for Women, Infants and Children. 
dent, we treated it as having a fixed effect at three points in time. We considered a categorical approach to data analysis, for which time was a variable (nonproportional hazard analysis). However, since some women had not completed breastfeeding, cutoff points (ages) for breastfeeding cessation were not clearly defined, and no precedence in the literature for this modeling approach was known $(8,24)$, we believed that the survival approach was more appropriate.

The WIC program is known to be successful at promoting breastfeeding, when mothers are enrolled as breastfeeding mothers, although success has been greater when women are encouraged to initiate breastfeeding than to continue breastfeeding $(8,11)$. The WIC program also decreases duration of breastfeeding when women are enrolled to receive formula. This issue deserves further study and policy consideration.

A mother's employment outside the home is a welldocumented correlate of weaning, although highly educated women often work and breastfeed. Nonetheless, urban, working mothers generally wean earlier, particularly in less developed countries $(25,26)$. No effect on duration of breastfeeding occurred if women worked part-time. Thus, in Hawaii the working circumstances of women employed full-time should be modified to promote longer breastfeeding duration.

Other reasons noted for weaning included not having enough milk, breast problems, and problems starting breastfeeding (9). Previous breastfeeding experience also promotes longer duration of breastfeeding $(3,7,9)$. It is expected that older women will breastfeed longer, since they are more mature, better able to postpone personal needs for those of the infant, and probably more confident (27).

Paid employment on rural islands may be more compatible with breastfeeding or other lifestyle characteristics particular to rural living, and may encourage longer breastfeeding. These women may provide models for interventions that could be used on urban Oahu. Reasons for longer breastfeeding duration among rural island women in Hawaii deserve further study.

Although the data in this study are 10 years old, they comprise the only data set in Hawaii that sampled infant feeding practices in a statewide sampling frame and included questions on breastfeeding initiation and cessation. In addition, the sample included ethnic groups that are not widely represented in other studies.

The response rate of 51 percent was low, but comparison with state vital statistics data of the same population showed the population who were missed in the study. Respondents were older than women in vital statistics data, with 18 percent 35 to 39 years old versus 8 percent in the state (State of Hawaii, Department of Health, 1987, unpublished data). Respondents were more educated than mothers in the state, with 24 percent who had graduated from college versus 12 percent in the state. The ethnic distribution was comparable to that in state data, except that Filipino women were underrepresented by 5 percent.

Maternal recall of breastfeeding may be biased, but previous studies of retrospective data on breastfeeding duration demonstrate the validity of these data (28).

Younger, less-educated, employed, local Japanese and some immigrant women in Hawaii require targeted programs to lengthen their duration of breastfeeding. Programs should address how and when to introduce foods, the use of formula, and management of paid employment and breastfeeding. Rural island breastfeeding women may provide models for overall management of breastfeeding in Hawaii.

\section{References}

1. U.S. Department of Health and Human Services. Healthy People 2000: National Health Promotion and Disease Prevention Objectives. DHHS Publication No. (PHS) 91-50213. Washington, DC: Author, 1991.

2. American Academy of Pediatrics. Breastfeeding and the use of human milk. Pediatrics 1997;100(6):1035-1039.

3. Kieffer EC, Novotny R, Welch KB, Mor JM, Thiele M. Health practitioners should consider parity when counseling mothers on infant feeding method decisions. J Am Dietet Assoc 1997; 97(11):1313-1316.

4. Goldberg D, Novotny R, Kieffer E, Mor J, Thiele M. Complementary feeding and ethnicity of infants in Hawaii. $J$ Am Dietet Assoc 1995;95(9):1029-1031.

5. Novotny R, Kieffer E, Mor J, Thiele M. Nativity and infant feeding pattern among Filipino women in Hawaii. Ecol Food Nutr 1995;33:263-272.

6. Novotny R, Kieffer E, Mor J, Thiele M, Nikaido M. Health of infant is the main reason for breastfeeding in a WIC population in Hawaii. J Am Dietet Assoc 1994;94(3):293-297.

7. Hill P, Humenick SS, Argubright TM, Aldag JC. Effects of parity and weaning practices on breastfeeding duration. Public Health Nurs 1997;14(4):227-234.

8. Gross SM, Caulfield LE, Bentley ME, et al. Counseling and motivational videotapes increase duration of breast-feeding in African-American WIC participants who initiate breastfeeding. J Am Dietet Assoc 1998;98:143-148.

9. Bourgoin GL, Lahaie NR, Rheaume BA, et al. Factors influencing the duration of breastfeeding in the Sudbury region. Rev Can Sante Publique 1997;88(4):238-241.

10. Piper S, Parks PL. Predicting the duration of lactation: Evidence from a national survey. Birth 1996;23(1):7-12.

11. Schwartz JB, Popkin BM, Tognetti J, Zohoori N. Does WIC participation improve breast-feeding practices? J Am Dietet Assoc 1995;95:729-731.

12. Novotny R, Kieffer E. Hawaii Infant Feeding Survey 1990 Honolulu: Hawaii State Department of Health, 1990.

13. SAS Institute. SAS Technical Report P-217, SAS/STAT Software: The PHREG Procedure, Version 6. Cary, NC: Author, 1991.

14. Cox DR. Regression models and life tables (with discussion). J Royal Stat Soc 1972;B4:187-220.

15. Abbott Laboratories, Ross Products Division. Mother's Survey. Columbus, OH: Author, 1995. 
16. Rodriguez-Garcia R, Frazier L. Cultural paradoxes relating to sexuality and breastfeeding. J Hum Lact 1995;11(2):111-115.

17. Greiner T. The concept of weaning: Definitions and their implications. J Hum Lact 1996;12(2):123-128.

18. Kordy MN, Ibrahim MA, El-Gamal FM, Bahnassy AA. Factors affecting duration of breastfeeding in a rural population of Saudi Arabia. Asia Pac J Public Health 1992/1993;6(1): $35-39$.

19. Hill PD, Humenick SS, Brennan M, Woolley D. Does early supplementation affect long-term breastfeeding? Clin Pediatr 1997;36(3):345-350.

20. Hill PD. Predictors of breast-feeding duration among WIC and non-WIC mothers. Public Health Nurs 1991;8(1):46-52.

21. Wright A, Rice S, Wells S. Changing hospital practices to increase the duration of breastfeeding. Pediatrics 1996;97(5): 669-675.

22. Righard L, Alade MO. Breastfeeding and the use of pacifiers. Birth 1997;24(2):116-120.
23. Lawrence R. Breastfeeding: A Guide for the Medical Profession. 4th ed. St Louis: Mosby-Year Book, 1994.

24. Pande H, Unwin C, Haheim LL. Factors associated with the duration of breastfeeding: Analysis of the primary and secondary responders to a self-completed questionnaire. Acta Paediatr 1997;86:173-177.

25. Huffman SL, Rasmussen E, Newman V, O'Gara C. Breastfeeding: A Natural Resource for Food Security San Diego: Wellstart International, 1992.

26. Swenson IE, Thang NM, Thieu PX. Individual and community characteristics influencing breastfeeding duration in Vietnam. Ann Hum Biol 1993;20(4):325-334.

27. O'Campo R, Faden RR, Gielen AC, Wang MC. Prenatal factors associated with breastfeeding duration: Recommendations for prenatal interventions. Birth 1992;19(4):195-201.

28. Holland B. The validity of retrospective breast-feeding data: An illustrative analysis of data quality in the Malaysian Family Life Survey. Hum Biol 1987;59(3):477-487. 